\title{
Lyapunov-Based PD Linear Control of the Oscillatory Behavior of a Nonlinear Mechanical System: The Inverted Physical Pendulum with Moving Mass Case
}

\author{
Carlos Fernando Aguilar-Ibáñez, ${ }^{1}$ \\ Oscar Octavio Gutiérrez-Frías, ${ }^{1}$ Juan Carlos Martínez-García, ${ }^{2}$ \\ Rubén Garrido-Moctezuma, ${ }^{2}$ and Bernardo Gómez-González ${ }^{3}$ \\ ${ }^{1}$ Centro de Investigación en Computación, Instituto Politécnico Nacional, \\ A. P. 75-476, 07700 México, DF, Mexico \\ ${ }^{2}$ Departamento de Control Automático, Instituto Politécnico Nacional, \\ A. P. 14-740, 07300 México, DF, Mexico \\ ${ }^{3}$ CANDE-INGENIEROS, Clemente Orozco No. 18, 03710 México, DF, Mexico \\ Correspondence should be addressed to Juan Carlos Martínez-García, \\ juancarlos.martinezg@gmail.com
}

Received 2 December 2009; Accepted 3 April 2010

Academic Editor: Oleg V. Gendelman

Copyright (c) 2010 Carlos Fernando Aguilar-Ibáñez et al. This is an open access article distributed under the Creative Commons Attribution License, which permits unrestricted use, distribution, and reproduction in any medium, provided the original work is properly cited.

This paper concerns active vibration damping of a frictionless physical inverted pendulum with a radially moving mass. The motion of the inverted pendulum is restricted to an admissible set. The proposed Proportional Derivative linear controller damps the inverted pendulum (which is anchored by a torsion spring to keep it in a stable upright position), exerting a force on the radially moving mass. The controller design procedure, which follows a traditional Lyapunovbased approach, tailors the energy behavior of the system described in Euler-Lagrange terms.

\section{Introduction}

Vibrating mechanical systems constitute an important class of dynamical systems. In fact buildings, bridges, car suspensions, pacemakers, wind generators, and hi-fi speakers (or even the mammalian middle ear) are common examples of this type of systems. In physical terms all vibrating systems consist of an interplay between an energy-storing component and an energy-carrying component. Thus, the behavior of the system can be described in terms of energy changes, that is, the motion of the system results from energy conversions. 
For theoretical and technological reasons, the control of vibrating mechanical systems is an important domain of research, which has provided technological solutions to several problems concerning oscillatory behaviors of some important classes of dynamical systems, for example, active control of vibrations is applied to attenuate undesired oscillations in buildings affected by external forces such as strong winds and earthquakes (see, e.g., [15] and the references therein), and computer-based active suspension is now common in cars as a means to improve road handling. It must be pointed out that in these examples control pursuits the elimination of the oscillatory behavior; however, in some applications the control purpose is to make the system vibrate in a convenient way (e.g., mechanical vibrations have been considered a potential choice for power-harvesting technologies for low-power electronic devices like MEMS; see, e.g., [6]).

As far as mathematical tools are concerned, the control of vibrations has mainly been tackled via frequency-domain techniques, which are essentially restricted to linear systems (see, e.g., $[7,8]$ ). When the vibrating systems are nonlinear, or when they oscillate too far away from their equilibrium points, frequency-domain techniques are not suitable. In the case of nonlinear systems characterized by small domains of attraction of the equilibrium points, the linear approach is not very effective. Hence, modern approaches prefer to follow time-domain nonlinear control strategies, which lie in ordinary differential equations, when lumped systems are concerned.

This paper focuses on active control of underdamped lumped nonlinear underactuated vibrating mechanical systems following an Energy-based approach, that is, the control of the vibratory behavior is tackled via the shaping of the energy flow which characterizes the system in dynamical terms. The control of vibrations is then considered in terms of the solution of a particular asymptotic stabilizing feedback control problem, around a selected equilibrium point. A stabilizing controller is then obtained following an Energybased Lyapunov approach, which exploits the physical properties of the involved mechanical system. In this way conservative control strategies based either on high gains or on cancelled nonlinear terms are avoided. It must be pointed out that standard nonlinear strategies such as sliding modes control and feedback linearization are frequently characterized by conservativeness (see, e.g., $[9,10])$. Intuitively speaking, Energy-based Lyapunov control shapes, via feedback control, the potential and kinetic energies of the controlled system in order to ensure a motion which guarantees the control objective (see, e.g., [11]). This approach requires the total energy of the concerned system to be a nonincreasing function. Moreover, the total energy function is also required to be at least locally positive definite around the selected equilibrium point (see, e.g., [5, 12-14]).

The main objective of this paper is to propose an asymptotic stabilizing controller, for the active vibration damping in a nonlinear underactuated and frictionless mechanical system, only which is restricted to move inside a predefined admissible set. This nonlinear mechanical system consists of a physical inverted pendulum, which rotates around a pivot located in the lower end of the pendulum arm, and an actuated auxiliary mass that moves, forward and backward, along of the pendulum arm. To keep the structure in the stable upright position (but not asymptotically stable) a restoring torsion spring is included.

The rest of the paper is organized as follows. Section 2 deals with the mathematical characterization of the dynamic behavior of the concerned system. The nonlinear mathematical model of the pendulum is obtained via the Euler-Lagrange approach. The proposed Proportional Derivative (PD) linear control law is exposed in Section 3, while Section 4 deals with some simulation of the closed-loop system. The paper concludes with some final comments in Section 5. 


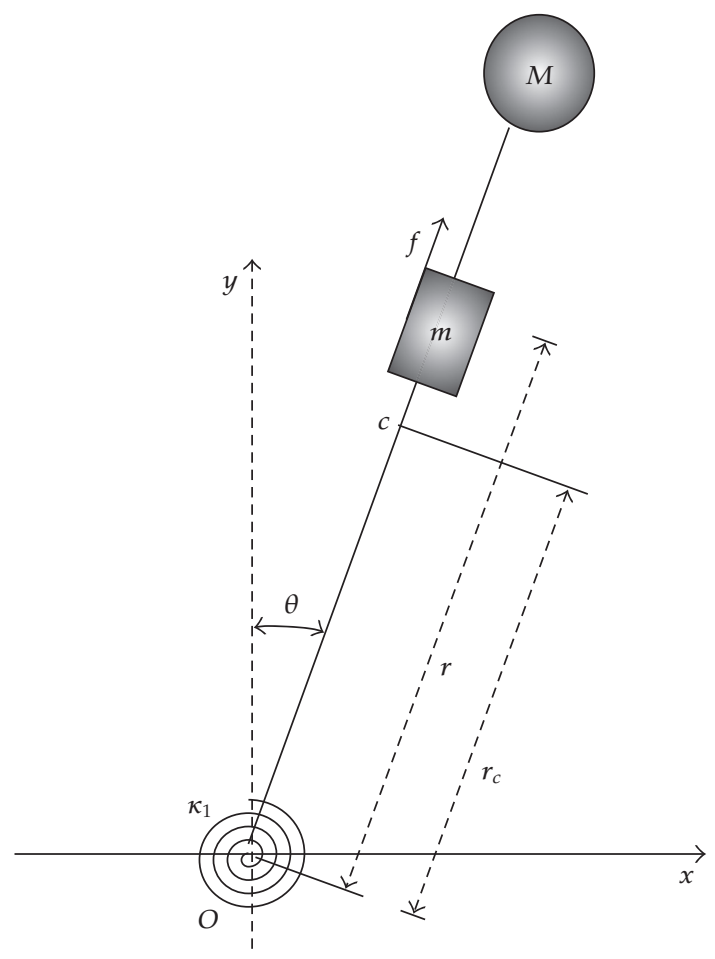

Figure 1: Inverted pendulum with a radially moving mass. The pendulum is anchored to the pivot point using a restoring torsion spring, which maintains the system in a stable upright position (but not asymptotically stable).

\section{Equation of Motion}

The dynamic system consists of a physical pendulum of mass $M$, which rotates about its pivot point $O$. Along the arm of the pendulum there is an auxiliary mass $m$ that can slide towards or away from the pivot. To keep the structure in a stable upright position a restoring moment is produced by a torsion spring with the spring constant denoted by $\kappa_{1}$, as we can see in Figure 1 (the system is assumed to be stable in the Lyapunov sense). The moment of inertia of the pendulum about the pivot is given by $I_{0}$, and its center of mass $C$ is located at the distance $r_{c}$ from the pivot. The mass $m$ is moved by a force $f$ applied on a direction parallel to OC. That is, $f$ is the control input that acts directly on the mass $m$.

In order to describe the pendulum motion, the origin of the inertial frame is chosen at point $O$. The $x$-axis and $y$-axis are set in the horizontal and vertical directions, respectively. Let the generalized coordinates be denoted by a two-dimensional vector $q:=[r, \theta]^{T}$ where $r$ is the radial displacement of the mass $m$ measured from the fixed pivot $O$, and $\theta$ denotes the angle formed by the $y$-axis and $\overline{O C}$. It is easy to show that both the total kinetic energy $K_{c}$ and the total potential energy $K_{p}$ of the system are given by

$$
\begin{gathered}
K_{c}=\frac{1}{2} I_{0} \dot{\theta}^{2}+\frac{1}{2} m\left(\dot{r}^{2}+r^{2} \dot{\theta}^{2}\right), \\
K_{p}=M g r_{c}(\cos \theta-1)+m g r \cos \theta+\frac{\kappa_{1}}{2} \theta^{2},
\end{gathered}
$$


respectively. Thus, the Lagrangian function $L(q, \dot{q})$ is given by

$$
L(q, \dot{q})=K_{c}-K_{p}
$$

Therefore, the corresponding Euler-Lagrange equations, that is, $(d / d t)((\partial L / \partial \dot{q})(q$, $\dot{q}))-(\partial L / \partial q)(q, \dot{q})=Q$, are given by

$$
\begin{gathered}
m \ddot{r}-m r \dot{\theta}^{2}+m g \cos \theta=F, \\
\left(m r^{2}+I_{0}\right) \ddot{\theta}+2 m r \dot{r} \dot{\theta}-g\left(M r_{c}+m r\right) \sin \theta+\kappa_{1} \theta=0,
\end{gathered}
$$

with the vector corresponding to the external forces being $Q=[F, 0]^{T}$. After applying the following feedback:

$$
F=m g+v
$$

into system (2.3), we can express the above set of differential equations as

$$
M(q) \ddot{q}+C(q, q) \dot{q}+\nabla_{q} K_{i}(q)=G v,
$$

where

$$
\begin{gathered}
M(q)=\left[\begin{array}{cc}
m & 0 \\
0 & m r^{2}+I_{0}
\end{array}\right], \quad C(q, \dot{q})=\left[\begin{array}{cc}
0 & -m r \dot{\theta} \\
m r \dot{\theta} & m r \dot{r} .
\end{array}\right], \quad G=\left[\begin{array}{l}
1 \\
0
\end{array}\right], \\
K_{i}(q):=-\kappa_{3}(1-\cos \theta)-\kappa_{2} r(1-\cos \theta)+\frac{\kappa_{1}}{2} \theta^{2} .
\end{gathered}
$$

Here $\kappa_{2}:=m g$ and $\kappa_{3}:=M g r_{c}$.

It is quite obvious that system (2.5) satisfies the followings properties:

(P1) $M(q)$ is positive definite;

(P2) matrix $H:=\dot{M}(q)-2 C(q, \dot{q})$ is a skew-matrix given by:

$$
H=\left[\begin{array}{cc}
0 & -m r \dot{\theta} \\
m r \dot{\theta} & 0
\end{array}\right],
$$

that is, $z^{T} H z=0$ for any $z \in \mathbb{R}^{2}$;

(P3) The operator $v \rightarrow \dot{r}$ is passive, since the time derivative of the total stored energy function $E(q, \dot{q})=(1 / 2) \dot{q}^{T} M(q) \dot{q}+K_{i}(q)$ is given after using the mentioned properties that $\dot{E}=v \dot{r}$.

Remark 2.1. Note that, if $v=0, \theta \in(-\pi / 2, \pi / 2)$ and $r>0$, then system (2.5) has a set of equilibrium points defined by $(r=\bar{r}, \theta=0, \dot{r}=0, \dot{\theta}=0)$, where $\bar{r}$ is a positive constant. These points are stable in the sense of Lyapunov but they are not asymptotically stable. 
In what follows we use the symbols $x$ and $\bar{x}$ to denote

$$
x=(q, \dot{q})=(r, \theta, \dot{r}, \dot{\theta}), \quad \bar{x}=(\bar{q}, 0)=(\bar{r}, 0,0,0)
$$

with $r>0$. On the other hand we use $z_{0}$ to indicate $z(0)$.

\section{PD Linear Control}

The above system is an underactuated and poorly damped mechanical system, since it has two degrees of freedom and it does not have one dissipative force in the nonactuated coordinate $\theta$. So that, this system is very sensible to external perturbations. In order to attenuate the undesirable effect of the external perturbations, we propose a stabilizing controller that makes the closed-loop system asymptotically stable around the origin $\bar{x}$.

Before establishing the control objective we introduce a necessary assumption:

(A1) the structural parameters of the original system satisfy the following relation:

$$
\kappa_{1}>\kappa_{3}+\kappa_{2}(\bar{r}+\varepsilon) .
$$

Remark 3.1. The inequality (3.1) means that the force produced by the spring is greater than the gravity force produced over the system, for any position of $m$. That is, (A1) is a structural condition related to the internal rigidities of the system.

The control objective is then posed as follows.

Problem 3.2. Find a smooth feedback $v$ that forces the system (2.5) to be asymptotically stable around the equilibrium point $\bar{x}$, restricted to move inside of an admissible set $Q \subset R^{2}$, defined by

$$
Q=\{q=(r>0, \theta):|r-\bar{r}|<\varepsilon,|\theta|<\bar{\theta}\}
$$

where $\varepsilon, \bar{\theta}$, and $\bar{r}$ are strictly positive constant, with $\bar{\theta}<\pi / 2$.

Remark 3.3. We must emphasize that the physical restrictions included in the formulation of the control problem are necessary to guarantee that the inverted pendulum can only moves inside a fraction of the upper half plane while the mass $m$ remains on the arm of the pendulum. That is, the auxiliary mass $m$ has to move along the pendulum length, and the angular position of the pendulum is restricted to move inside of a given interval defined by $(-\bar{\theta}, \bar{\theta})$. In other words, we ask for the auxiliary mass $m$ to move along the pendulum arm and at the same time we ask the pendulum angular displacement to be confined to a vicinity near to the vertical top position.

In what follows we tackle the solution of the stabilizing feedback control problem. 


\subsection{Energy-Based Control}

Consider the following candidate Lyapunov function:

$$
E_{T}(x)=E_{T}(q, \dot{q})=\frac{1}{2} \dot{q}^{T} M(q) \dot{q}+K_{m}(q),
$$

where $K_{m}(q)$ is the modified potential energy stated as

$$
K_{m}(q)=K_{i}(q)+\frac{k_{p}}{2}(r-\bar{r})^{2}
$$

with $k_{p}>0$.

Remark 3.4. Under assumption (A1), the modified potential energy $K_{m}(q)$ has a local minimum at $\bar{q}=(\bar{r}, 0)$. This follows from the fact that

$$
K_{m}(\bar{q})=0, \quad \nabla_{q} K_{m}(\bar{q})=0, \quad \nabla_{q}^{2} K_{m}(\bar{q})>0
$$

That is, $K_{m}(q)$ is a convex function around $\bar{q}$. Hence, the level curves of $K_{m}(q)$ are constituted by a set of closed-loop curves around $\bar{q}$. This property allows us to define a compact invariant set, that we will use for the convergence analysis.

Taking into account the passivity properties of system (2.5), the first time derivative of $E_{T}$ along the trajectories of the system is given by

$$
\dot{E}_{T}(q, \dot{q})=v \dot{r}+k_{p}(r-\bar{r}) \dot{r}
$$

Since this derivative needs to be definite negative, the following Proportional Derivative linear control law is proposed:

$$
v=-k_{p}(r-\bar{r})-k_{d} \dot{r}
$$

which leads to

$$
\dot{E}_{T}(q, \dot{q})=-k_{d} \dot{r}^{2}
$$

with $k_{d}>0$.

As $E_{T}$ is strictly positive definite and $\dot{E}_{T}$ is negative semidefinite, we can conclude the stability of the closed-loop system in the Lyapunov sense. That is, $q$ and $\dot{q}$ are bounded. To ensure that the closed-loop solution asymptotically converges to the origin $\bar{x}$ we need to use the LaSalle invariance theorem.

Before applying the well-known LaSalle invariance theorem (see, e.g., [15]), we need to introduce a useful Lemma which allows us to select the constant $k_{p}$ provided that all the solutions of the obtained closed-loop system remain inside of the admissible set $Q$. 
Lemma 3.5. Consider the closed-loop system (see (2.5) and (3.7)). Under assumption (A1) and the restriction of parameter $k_{p}$, given by

$$
\frac{\kappa_{2}}{k_{p}}(1-\cos \bar{\theta})<\varepsilon
$$

if the initial conditions $x_{0}=\left(q_{0}, \dot{q}_{0}\right)$ with $q_{0} \in Q$, satisfying:

$$
E_{T}\left(x_{0}\right) \leq \bar{E}
$$

where the bound $\bar{E}$ is defined as

$$
\bar{E}=\max \left\{c>0: K_{m}(q)=c \text {, with } q \in Q\right\}
$$

and can be estimated solving equality

$$
\bar{E}=K_{m}\left(\bar{r}+\frac{\varepsilon}{\lambda^{\prime}}, \bar{\theta}\right)=\lambda \varepsilon \kappa_{2}(1-\cos \bar{\theta}),
$$

where $\lambda>1$, then one guarantees that

$$
E_{T}(q(t), \dot{q}(t)) \leq E_{T}\left(x_{0}\right) \leq \bar{E},
$$

with $q(t) \in Q, t \geq 0$ (see the appendix). set $\Omega$

Now we are ready to apply the LaSalle invariance theorem. Let us define a compact

$$
\Omega=\left\{x=(q, \dot{q}): E_{T}(x)<\bar{E}\right\}
$$

where $\bar{E}>0$ is selected according to Lemma 3.5.

Remark 3.6. The set $\Omega$ has the property that all the solutions of the closed-loop system that start in $\Omega$ remain in $\Omega$ for all future time. In particular, all initial conditions $x_{0}=\left(q_{0}, \dot{q}_{0}\right)$ such that $E_{T}\left(x_{0}\right)<\bar{E}$, with $q_{0} \in Q$ imply that $q(t) \in Q$, for all future.

Let $\Gamma$ be defined as follows:

$$
\Gamma=\left\{x \in \Omega: \dot{E}_{T}(x)=0\right\}=\{x \in \Omega: \dot{r}=0\},
$$

and let $\underline{M}$ be the largest invariant set in $\Gamma$. The LaSalle invariance theorem guarantees that every solution starting in a compact set $\Omega$ approaches $\underline{M}$ as $t \rightarrow \infty$ [15]. Let us then compute the largest invariant set $\underline{M}$ in $\Gamma$. On the set $\Gamma$, we have that $\ddot{r}=0$ and $r=\underline{r}$, where $\underline{r}$ is a fixed constant, such that $|\bar{r}-\underline{r}|<\varepsilon$ (for simplicity, we use the symbol $\underline{x}$ to denote that $(d / d t) \underline{x}=$ $0)$. Suppose that in $\Gamma, r \neq \underline{r}$. Then, from the definition of $v(3.7)$, we have that $v=-k_{p}(\underline{r}-\bar{r}) \neq 0$, 
which leads to a contradiction since a constant force will eventually produce a displacement of the auxiliary mass $m$ and state $r$ cannot be bounded (see first equation of (2.5)). Hence, in the set $\Gamma, r=\bar{r}$. Thus, in the set $\Gamma$ system (2.5) becomes

$$
\begin{gathered}
-m \bar{r} \dot{\theta}^{2}+\kappa_{2}(\cos \theta-1)=0 \\
\left(m \bar{r}^{2}+I_{0}\right) \ddot{\theta}-\left(\kappa_{3}+\kappa_{2} \bar{r}\right) \sin \theta+\kappa_{1} \theta=0 .
\end{gathered}
$$

From the two previous equations we must have that the single trajectory that satisfies (3.16) is given by $\dot{\theta}$ and $\theta$ which are equal to zero in the set $\Gamma$, because $|\theta|<\bar{\theta}<\pi / 2, \kappa_{2}>0, \bar{r}>0$. Therefore, we concluded that the largest invariant set contained in $\Gamma \subset \Omega$, that is, $M$, is given by the single equilibrium point $\bar{x}=(r=\bar{r}, \theta=0, \dot{r}=0$, and $\dot{\theta}=0)$. According to the LaSalle invariance theorem, all trajectories that start in $\Omega$ asymptotically converge towards the largest invariant set $\underline{M}$, which is given by the single point $\bar{x}$.

This section concludes with the following proposition.

Proposition 3.7. Under the assumptions of Lemma 3.5. Let the system (2.3) in closed loop, with

$$
F=m g-k_{p}(r-\bar{r})-k_{d} \dot{r}
$$

Then the origin of the closed-loop system is locally asymptotically stable with a computable domain of attraction defined by the inequality (3.13). Besides the closed-loop solution is restricted to move inside of the admissible set $Q$.

Remark 3.8. It is easy to check that if assumption (A1) is relaxed, we can assure asymptotic stability of the closed-loop system. However, we cannot assure that $x(t)$ belongs to $Q$, for all $t>0$. On the other hand, if the physical parameters do not satisfy (A1), we then have three equilibrium points, and we only can assure stability in the Lyapunov sense.

In order to illustrate the proposed energy-based feedback (PD linear) control law, we perform in the following section some computer-based simulations.

\section{Simulations}

\subsection{Simulation Settings}

In order to carry out the simulation of the closed-loop system, we set the system parameters to be

$$
\begin{gathered}
m=1(\mathrm{~kg}), \quad M=4(\mathrm{~kg}), \quad I_{0}=0.5\left(\mathrm{~kg} \cdot \mathrm{m}^{2}\right), \quad r_{c}=0.5(\mathrm{~m}), \\
\bar{r}=0.5(\mathrm{~m}), \quad \varepsilon=0.45(\mathrm{~m}), \quad \bar{\theta}=0.8(\mathrm{rad}), \quad \kappa_{1}=31.25 .
\end{gathered}
$$

Of course, in this case $\kappa_{2}=9.8$ and $\kappa_{3}=19.6$, and, evidently, the physical system satisfies the structural assumption (A1). 

produces

The parameter value of $k_{p}$ has to be chosen according to restriction (3.9), which

$$
7.34<k_{p}
$$

Note that the admissible set is given by

$$
Q=\{(r>0, \theta):|r-0.5|<0.4,|\theta|<0.8\} .
$$

To ensure that the initial condition vector is within the maximal domain of attraction, we set the parameters $\lambda=4.1$ and $k_{p}=30.46$ according to relations (3.12) and (A.5), respectively. Hence, to maintain the closed-loop motion inside the domain of attraction the condition given by $E_{T}\left(q_{0}, \dot{q}_{0}\right)<\bar{E} \cong 2.43$, with $q_{0} \in Q$, must be satisfied.

\subsection{Comments on the Simulations}

We simulate the closed-loop behavior of the nonlinear mechanical system using the Matlab + Simulink ${ }^{\mathrm{TM}}$ computational platform.

We choose as the initial conditions vector $q_{0}=(0.6(\mathrm{~m}), 0.8(\mathrm{rad}))$, with zero velocity states, satisfying $E_{T}\left(q_{0}, 0\right)=2.36$ (vector $q_{0}$ is selected very close to one of the extreme points in the direction of $\theta$ that belong to $\partial Q$, the set of all boundary points of $Q$ ). Figure 2 shows the transient behavior of the position and the velocity variables resulting from the chosen initial conditions vector. As we can see the position variables are inside of set $Q$, since the initial conditions vector belongs to the domain of attraction of the closed-loop system.

When choosing $x_{0}=(0.7(\mathrm{~m}), 0.6(\mathrm{rad}), 0,2(\mathrm{rad} / \mathrm{s}))$ with $E_{T}\left(x_{0}\right)=3.75>\bar{E}$ (i.e., $q$ may go out of $Q$ ), we obtain the behavior shown in Figure 3. In this particular case, even when the initial conditions vector belongs to the admissible set $Q$, the closed-loop responses are outside of the restricted set $Q$ since $E_{T}\left(x_{0}\right)>\bar{E}$ (see that $\theta \simeq 1(\mathrm{rad})$, when $t=2(\mathrm{~s})$ ).

\section{Concluding Remarks}

In this work, we presented a Lyapunov-based approach for the asymptotic stabilization of a frictionless inverted physical pendulum (which is maintained in the stable upright position, in the Lyapunov sense, via the inclusion of a torsion spring which anchors the pendulum to the pivot) with a radially moving mass. The motion of the pendulum is restricted to be in an admissible set $Q$ (3.2), which characterizes physical restrictions. The proposed Proportional Derivative control strategy exploits the underlying physical properties of the original system, which have been used to shape the total energy of the closed-loop system.

The stability analysis of the closed-loop system has been carried out by using the well-known LaSalle invariance theorem. It is worth mentioning that if a damping force is considered in the nonactuated coordinate then asymptotically stability of this device is reinforced.

Concerning the applicability of the proposed control law, the nonlinear mechanical system chosen here models, in a simplified way, the dynamics of rigid buildings restricted to oscillate in the plane (when affected by external excitations). We are interested in the attenuation of the effects of unknown disturbances (seismic forces) on the behavior of civil 

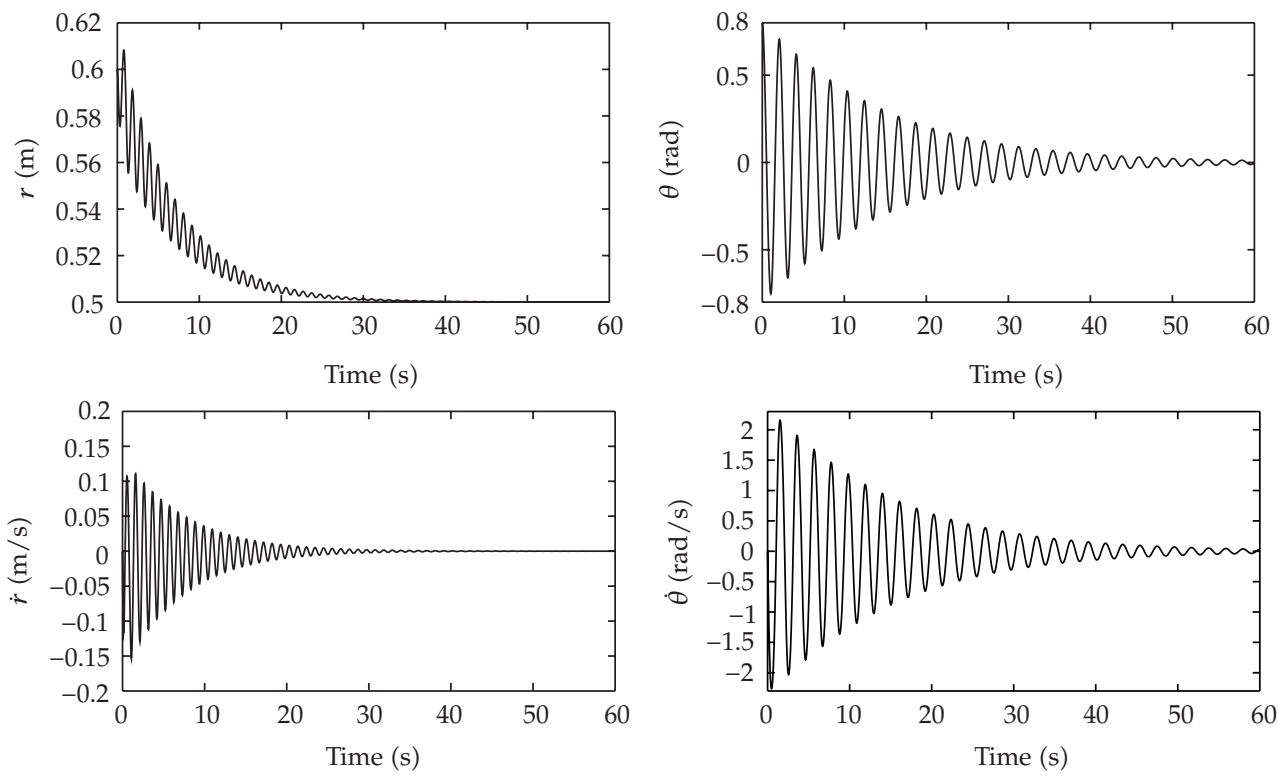

Figure 2: Simulation of the closed-loop system starting from $q(0)=(0.6,0.8)$ and $\dot{q}(0)=0$.
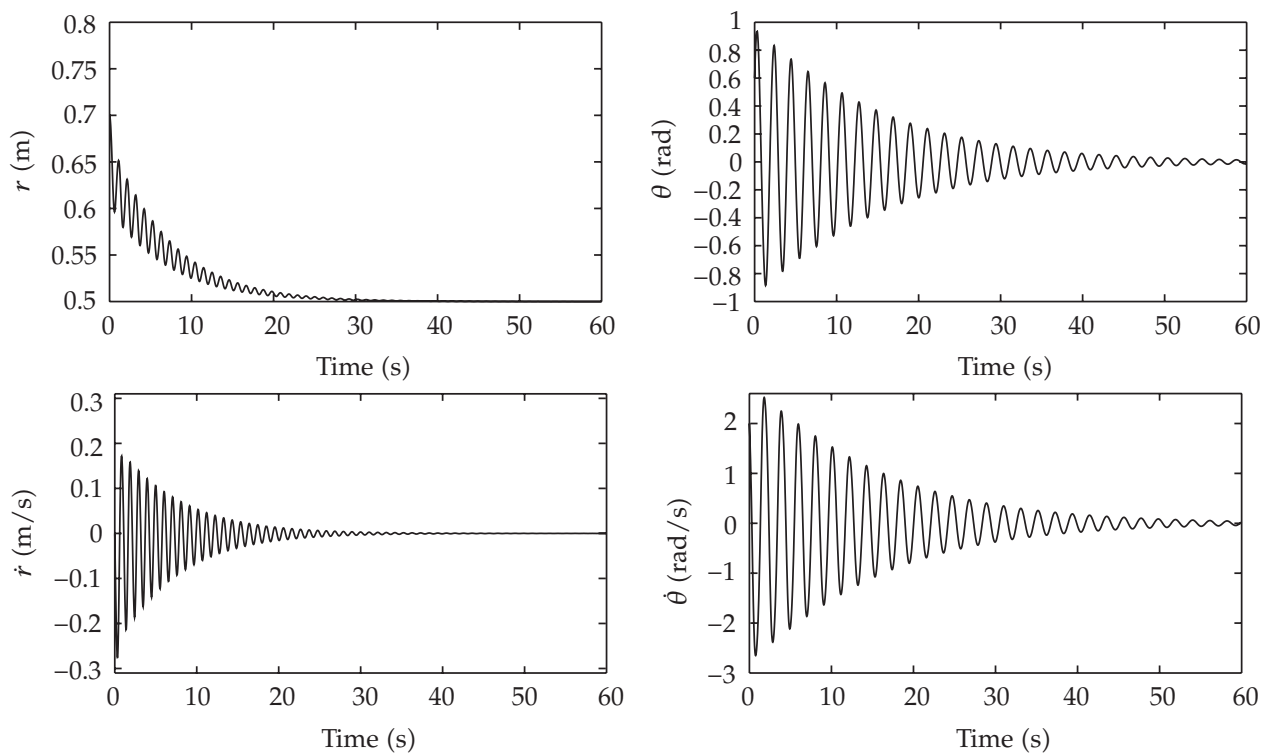

Figure 3: Closed-loop response of the nonlinear system, when the initial condition is stated as $x_{0}=$ $(0.7,0.6,0,2)$.

structures via smooth active control. In the considered model the radially moving mass is proposed as an active control element; the potentiality of such an actuator must be clarified via the evaluation of the energy consumption characteristics of the control law. 


\section{Appendix}

Proof of Lemma 3.5. In the fist part we estimate the bound $\bar{E}$, defined in (3.11). The idea behind it is to build the largest level curve contained in the admissible set $Q$.

First of all we define the set $S_{c}$ as follows:

$$
S_{c}:=\left\{q \in Q: K_{m}(q) \leq c>0\right\} .
$$

Note that from assumption (A1) and Remark 3.6, $S_{c}$ is a convex set. Computing the extreme points in the direction of $\theta$ and $r$ of the set $S_{c}$, we have the following relations:

$$
\begin{gathered}
\frac{\partial K_{m}}{\partial \theta}(q)=-\left(\kappa_{3}+\kappa_{2} r\right) \sin \theta+\kappa_{1} \theta=0 \\
\frac{\partial K_{m}}{\partial r}(q)=-\kappa_{2}(1-\cos \theta)+k_{p}(r-\bar{r})=0 .
\end{gathered}
$$

From (A1) we conclude that the single solution of (A.2) is given by $\theta=0$. Consequently, the extreme points in the direction of $\theta$ are given by $q_{1}=(\bar{r}+\varepsilon, 0)$ and $q_{2}=(\bar{r}-\varepsilon, 0)$. If we desire that $q_{1}$ and $q_{2}$ belong to $\partial Q$, then we must have that $K_{m}\left(q_{1}\right)=$ $K_{m}\left(q_{2}\right)=k_{p} \varepsilon^{2} / 2(\partial Q$ denotes the set of all boundary points of $Q)$. Analogously, the other extreme points in the direction of $r$ that belong to $\partial Q$ are given by $q_{3}=(\bar{r}+\varepsilon / \lambda, \bar{\theta})$ and $q_{4}=(\bar{r}+\varepsilon / \lambda, \bar{\theta})$, where the parameter $\lambda$ is selected as (note that in order to guarantee that $q_{3}$ and $q_{4}$ belong to $\partial Q$ we must have that $\lambda>1$.)

$$
K_{m}\left(q_{3}\right)=K_{m}\left(q_{4}\right)=\lambda k_{2}(1-\cos \bar{\theta}) \varepsilon=\frac{k_{p}}{2} \varepsilon^{2} .
$$

Indeed, after solving (A.3) and forcing that the two solutions belong to $\partial Q$, it follows that $q=\left(r_{*}, \pm \bar{\theta}\right) \in \partial Q$, where $r_{*}=\bar{r}+\kappa_{2}\left(1-\cos \theta_{*}\right) / k_{p}$. Thus, defining $k_{p}$ as

$$
k_{p}=\frac{\lambda k_{2}(1-\cos \bar{\theta})}{\varepsilon},
$$

with $\lambda>1$ and $\bar{E}=k_{p} \varepsilon^{2} / 2$, we have that the values of $r_{*}$ and $E$ can be rewritten as

$$
r_{*}=\bar{r}+\frac{\varepsilon}{\lambda} ; \quad \bar{E}=\lambda k_{2}(1-\cos \bar{\theta}) \varepsilon
$$

And, evidently, there is a $\lambda>1$ satisfying (3.12).

Now, since $E_{T}$ is a nonincreasing function, it follows that if $x_{0}=\left(q_{0}, \dot{q}_{0}\right)$ with $q_{0} \in Q$ and $E_{T}\left(x_{0}\right) \leq \bar{E}$, then we have

$$
K_{m}(q(t)) \leq K_{m}(q(t))+\frac{1}{2} \dot{q}^{T}(t) M \dot{q}(t) \leq E_{T}(x(t)) \leq E_{T}\left(x_{0}\right) \leq \bar{E}, \quad \forall t \geq 0
$$


From the above inequality we have that $q(t) \in S_{\bar{E}} \subset Q$, for all $t \geq 0$. Hence, Lemma 3.5 is fulfilled.

\section{Acknowledgments}

This research was supported by the following mexican institutions: the Centro de Investigación en Computación of the Instituto Politécnico Nacional (CIC-IPN); the Coordinación de Posgrado e Investigación of the Instituto Politécnico Nacional, under Research Grant 20020247; the Centro de Investigación y Estudios Avanzados del Instituto Politécnico Nacional (Cinvestav-IPN); CONACYT-México, under Research Grant 32681-A.

\section{References}

[1] D. Inman, Engineering Vibration, Prentice Hall, New York, NY, USA, 1994.

[2] H. Matsuhisa, R. Gu, Y. Wang, O. Nishihara, and S. Sato, "Vibration control of a ropeway carrier by passive dynamic vibration absorbers," JSME International Journal, vol. 38, no. 4, pp. 657-662, 1995.

[3] P. Dong, H. Benaroya, and T. Wei, "Integrating experiments into an energy-based reduced-order model for vortex-induced-vibrations of a cylinder mounted as an inverted pendulum," Journal of Sound and Vibration, vol. 276, no. 1-2, pp. 45-63, 2004.

[4] H. Sira-Ramírez and O. Llanes-Santiago, "Sliding mode control of nonlinear mechanical vibrations," Journal of Dynamic Systems, Measurement and Control, vol. 122, no. 4, pp. 674-678, 2000.

[5] C. Aguilar-Ibañez and H. Sira-Ramirez, "PD control for active vibration damping in an underactuated nonlinear system," Asian Journal of Control, vol. 44, no. 4, pp. 502-508, 2002.

[6] S. Behrens, "Potential system efficiencies for MEMS vibration energy harvesting," in Smart Structures, Devices, and Systems III, S. F. Al-Sarawi, Ed., vol. 6414 of Proceedings of SPIE, Adelaide, Australia, 2007, 64140D.

[7] W. T. Thomson, Theory of Vibrations with Applications, Allen and Unwin, London, UK, 1981.

[8] C. Fuller, S. J. Elliot, and P. A. Nelson, Active Control of Vibration, Academic Press, San Diego, Calif, USA, 1996.

[9] V. Utkini, Sliding Modes Control in Electromechanical Systems, Taylor \& Francis, London, UK, 1999.

[10] A. Isidori, Nonlinear Control Systems, Communications and Control Engineering Series, Springer, New York, NY, USA, 3rd edition, 1995.

[11] R. Ortega, A. Loria, R. Nicklasson, and H. Sira-Ramirez, Passivity-Based Control of Euler-Lagrange Systems, Springer, Berlin, Germany, 1998.

[12] I. Fantoni and R. Lozano, Non-Linear Control for Underactuated Mechanical Systems, Springer, London, UK, 2002.

[13] C. Aguilar-Ibañez, O. Gutiérrez Frías, and M. Suárez Castañón, "Lyapunov based controller for the stabilization of the cart-pendulum system," Nonlinear Dynamics, vol. 40, no. 4, pp. 367-374, 2005.

[14] C. Aguilar-Ibañez and H. Sossa-Azuela, "Lyapunov based controller for the stabilization of the Furuta Pendulum," Nonlinear Dynamics, vol. 49, pp. 1-8, 2006.

[15] H. K. Khalil, Non-Linear Systems, Prentice Hall, Bergen, NJ, USA, 2nd edition, 1996. 


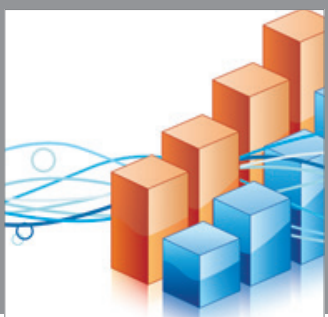

Advances in

Operations Research

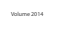

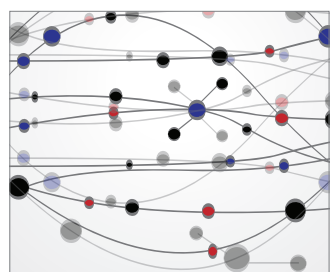

\section{The Scientific} World Journal
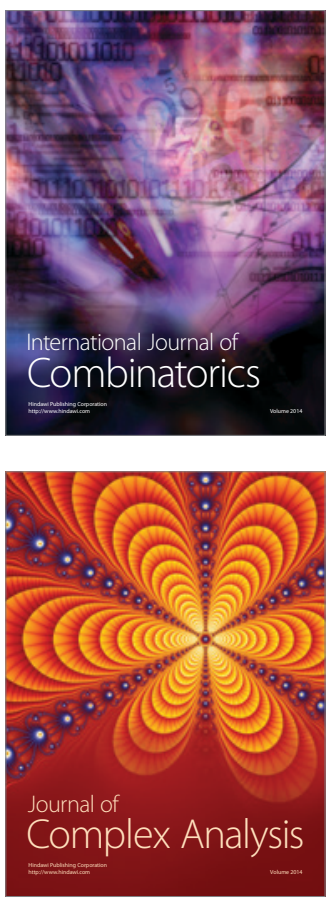

International Journal of

Mathematics and

Mathematical

Sciences
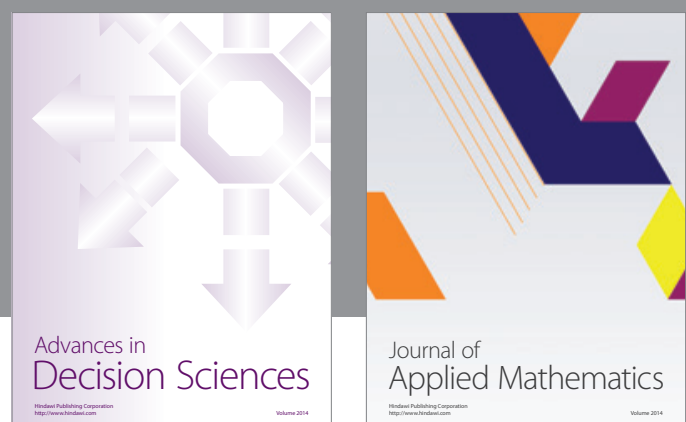

Journal of

Applied Mathematics
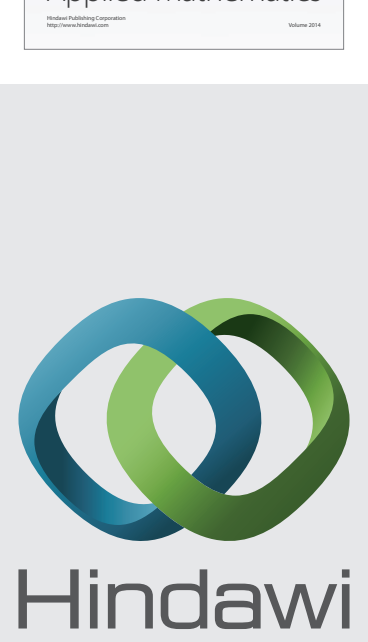

Submit your manuscripts at http://www.hindawi.com
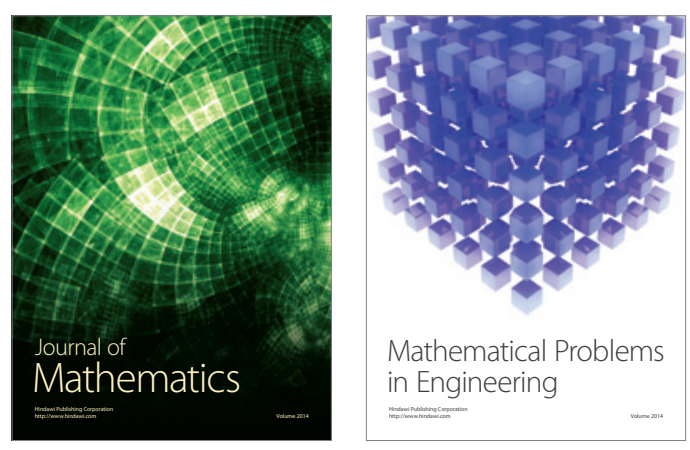

Mathematical Problems in Engineering
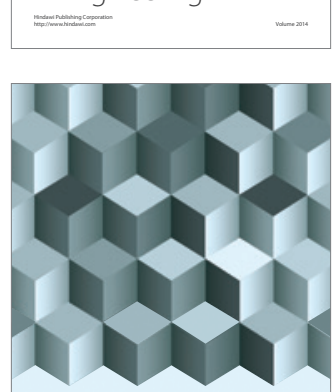

Journal of

Function Spaces
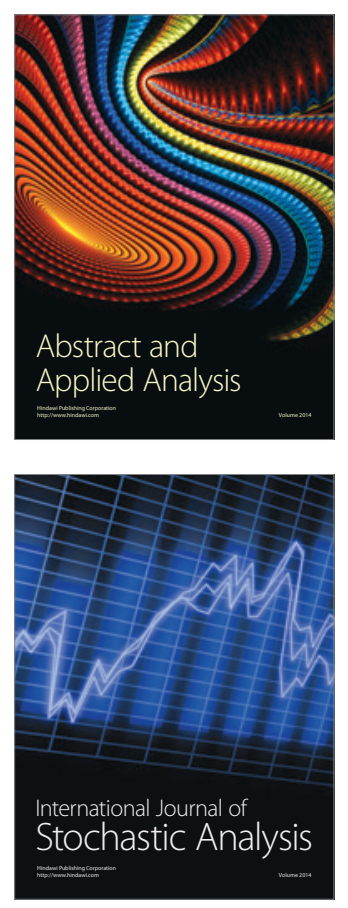

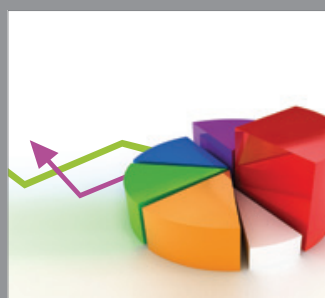

ournal of

Probability and Statistics

Promensencen
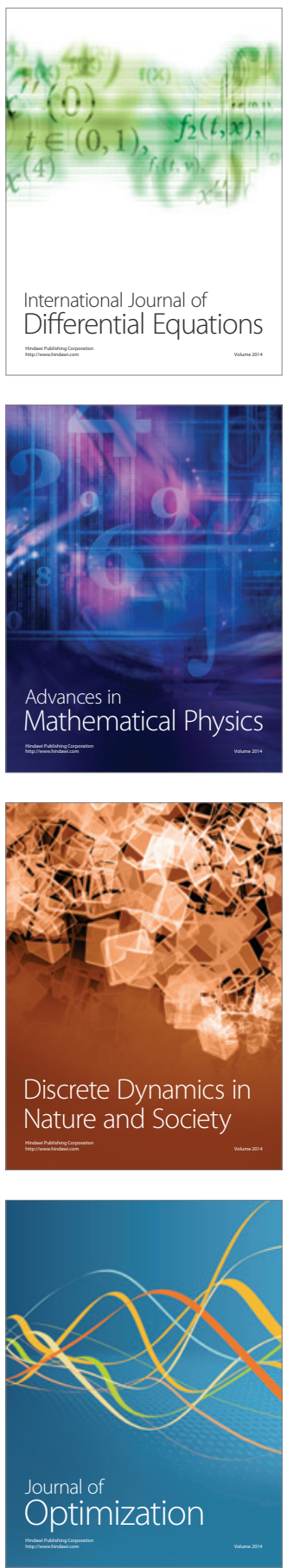